\title{
Physiological consequences of tropomyosin mutations associated with cardiac and skeletal myopathies
}

Received: 10 June 2000 / Accepted: 3 October 2000 / Published online: 15 November 2000

(C) Springer-Verlag 2000

Abstract Mutations have been identified in $\alpha$-tropomyosin $(\mathrm{Tm})$, a key regulatory protein in striated muscle cells, that are associated with a human cardiac myopathy, hypertrophic cardiomyopathy (FHC) and a human skeletal myopathy, nemaline myopathy (NM). In this review,

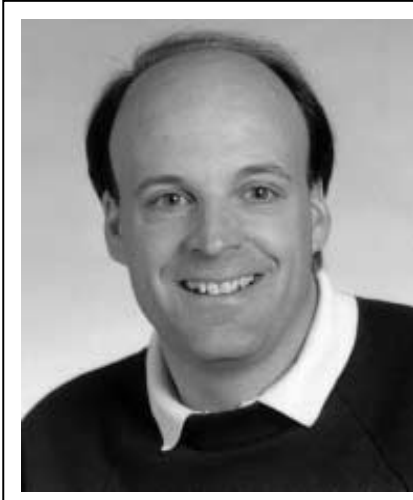

JOSEPH M. METZGER Joseph Metzger received his $\mathrm{Ph} . \mathrm{D}$. from Marquette University and post-doctoral training at the University of Wisconsin. $\mathrm{He}$ is currently Associate Professor of Physiology and Internal Medicine at the University of Michigan. His research is focused on genetic modification of cardiovascular function. His work features transgenesis and gene transfer approaches to re-design heart function in health and disease.

D.E. Michele · J.M. Metzger $(-)$

Department of Physiology, University of Michigan,

Ann Arbor, MI 48109, USA

e-mail: metzgerj@umich.edu

Tel.: +1-734-7635844, Fax: +1-734-9368813

J.M. Metzger

Department of Physiology, 7730 Medical Science II, University of Michigan, 1301 E. Catherine, Ann Arbor, MI 48109-0622, USA we highlight experiments aimed at identifying the underlying mechanisms by which mutations in $\alpha$-Tm cause inherited diseases of cardiac and skeletal muscle. Gene transfer of normal and mutant $\alpha$-Tm to isolated adult cardiac myocytes was used to study the primary effects of mutant $\alpha$-Tm proteins on the structure and contractile function of fully differentiated striated muscle cells. Both FHC and NM mutant $\alpha$-Tm proteins incorporated normally into the adult muscle sarcomere, similar to normal Tm but exerted differential "dominant-negative" effects on the contractile function of the muscle cell. FHC mutant $\alpha-T m$ proteins produced hypersensitivity of $\mathrm{Ca}^{2+}$-activated force production with a hierarchy that was related to the clinical severity of each mutation. Conversely, the NM mutant $\alpha$-Tm produced a hyposensitivity of $\mathrm{Ca}^{2+}$-activated force production that may underlie, at least in part, the muscle weakness observed in NM. Taken together, the results suggest that the differential changes in the ability of the mutant Tm proteins to regulate muscle contraction in response to changing $\mathrm{Ca}^{2+}$ concentrations underlie the differential clinical presentation of the cardiac and skeletal muscle myopathies associated with mutations in $\alpha$-Tm.

Keywords Calcium $\cdot$ Hypertrophic cardiomyopathy · Muscle contraction $\cdot$ Nemaline myopathy $\cdot$ Tropomyosin

The contractile apparatus of fully differentiated striated muscle, which includes both cardiac and skeletal muscle, is a complex array of proteins arranged in interdigitating thick and thin filaments and is designed to produce both force and motion. The overall structure and regulation of cardiac and skeletal muscle are generally similar. Depolarization of the striated muscle cell membrane leads to a transient release of $\mathrm{Ca}^{2+}$ from the internal $\mathrm{Ca}^{2+}$ stores of the sarcoplasmic reticulum. $\mathrm{Ca}^{2+}$ then binds to the troponin regulatory complex which initiates conformational changes in the thin filament, initiating muscle contraction. The troponin complex is composed of three sub- 
units. $\mathrm{Ca}^{2+}$ binds to the troponin $\mathrm{C}(\mathrm{TnC})$ subunit, which causes changes in the interactions of $\mathrm{TnC}$ and troponin I (TnI), relieving the inhibitory binding of troponin I to actin. The release of inhibition is transmitted through troponin $\mathrm{T}(\mathrm{TnT})$ to tropomyosin $(\mathrm{Tm})$, a coiled-coil dimer that coils around the actin filament and spans seven actin monomers for every troponin complex. Binding of $\mathrm{Ca}^{2+}$ to troponin allows the movement of Tm over the surface of the actin filament, which exposes stereospecific myosin binding sites on actin. Myosin can then bind strongly to actin and use ATP hydrolysis to drive both force production and muscle shortening (for review see $[1,2])$. Therefore, Tm plays an integral role in the regulation of muscle contraction, as it "relays" the information from the $\mathrm{Ca}^{2+}$ sensor, a single troponin complex, to the seven actin monomers and the actin/myosin interactions that produce force and motion.

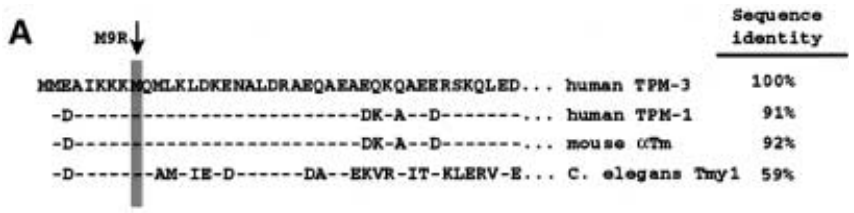

B

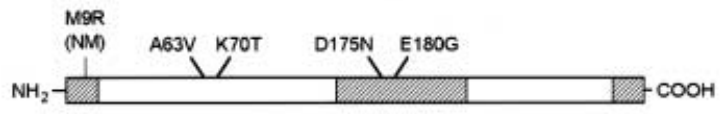

C

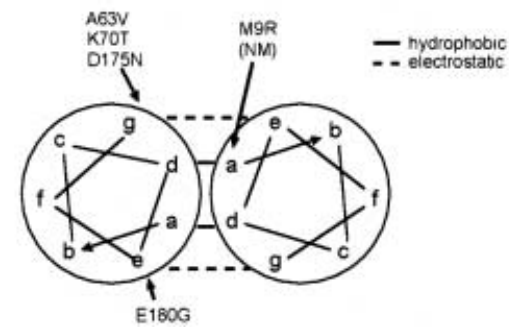

Fig. 1A-C Tropomyosin structure and mutations associated with hypertrophic cardiomyopathy and nemaline myopathy. A Alignment of alpha tropomyosin $(\alpha \mathrm{Tm})$ isoforms. Genebank accession numbers are: TPM3, X04201; TPM1, M19713; rat, SEG_RATTMA; C. e., D38540. B Position of Tm mutations in the primary structure of $\alpha-T m$. The shaded areas represent the putative regions in $\alpha-T m$ that bind troponin T (TnT) [17]. C Predicted alpha helical coiled-coil structure of $\alpha$-Tm. View is from the N-terminus of $\alpha$-Tm looking down the axis of the Tm dimer. The positions " $a-g$ " are the amino acids of the alpha-helical heptad repeat in $\alpha$ $\operatorname{Tm}[18]$

Table 1 Tropomyosin mutations and diseases of cardiac and skeletal muscle

\begin{tabular}{|c|c|c|c|c|c|}
\hline & Disease gene & Mutation & Inheritance & Phenotype & Reference \\
\hline FHC & $\begin{array}{l}\text { TPM } 1 \\
\text { TPM1 } \\
\text { TPM1 } \\
\text { TPM1 }\end{array}$ & $\begin{array}{l}\text { A63V } \\
\text { K70T } \\
\text { D175N } \\
\text { E180G }\end{array}$ & $\begin{array}{l}\text { Autosomal dominant } \\
\text { Autosomal dominant } \\
\text { Autosomal dominant } \\
\text { Autosomal dominant }\end{array}$ & $\begin{array}{l}\text { Cardiomyopathy } \\
\text { Cardiomyopathy } \\
\text { Cardiomyopathy } \\
\text { Cardiomyopathy }\end{array}$ & $\begin{array}{l}{[11,12]} \\
{[12]} \\
{[10,11,16]} \\
{[10,16]}\end{array}$ \\
\hline NM & $\begin{array}{l}\text { TPM3 } \\
\text { TPM3 } \\
\text { TPM1 }\end{array}$ & $\begin{array}{l}\text { M9R } \\
\text { 31stop } \\
\text { Candidate gene }\end{array}$ & $\begin{array}{l}\text { Autosomal dominant } \\
\text { Autosomal recessive } \\
\text { Autosomal recessive }\end{array}$ & $\begin{array}{l}\text { Adult-onset skeletal myopathy } \\
\text { Severe infantile skeletal myopathy } \\
\text { Severe infantile skeletal myopathy }\end{array}$ & $\begin{array}{l}{[23]} \\
{[28]} \\
{[29]}\end{array}$ \\
\hline
\end{tabular}

Multiple tropomyosin isoforms exist and are expressed in almost every mammalian cell type. These different isoforms result from the alternative splicing of four different Tm genes in humans [3]. In cardiac muscle of adult rodents and humans, Tm is composed exclusively of $\alpha$-Tm (TPM1 gene product) which is identical in sequence to that expressed in fast-twitch skeletal muscle. Embryonic cardiac muscle is composed primarily of Tm expressed from the $\beta$-Tm gene (TPM2). Thus, there is a developmental switch from $\beta$-Tm to $\alpha$-Tm during the developmental maturation of the adult heart. Adult skeletal muscle is composed of $\alpha-\mathrm{Tm}$ and $\beta$-Tm with the relative amounts of each being dependent on fiber type [4]. In slow skeletal muscle, the predominant $\alpha$-Tm isoform is expressed from the TPM3 gene. The comparison of the sequences of TPM1 and TPM3 Tm in Fig. 1A shows that the $\alpha-T m$ expressed from the TPM1 gene or the TPM3 gene shows a high degree of sequence identity and convergence, especially near the $\mathrm{N}$-terminus of $\alpha$-Tm. The TPM4 gene encodes a non-muscle Tm expressed in fibroblasts and platelets [3].

\section{Tropomyosin mutations and diseases of cardiac muscle: familial hypertrophic cardiomyopathy}

The key role of $\mathrm{Tm}$ in the regulation of striated muscle contraction has been highlighted by the identification of mutations in Tm genes that are associated with inherited human diseases of cardiac and skeletal muscle (Table 1). Familial hypertrophic cardiomyopathy (FHC) is an inherited disease of heart muscle characterized by abnormal cardiac hypertrophy in the absence of known systemic stimuli for cardiac hypertrophy $[5,6]$. In addition to cardiac hypertrophy, FHC often results in structural abnormalities of myocyte disarray, sarcomere disruption and interstitial fibrosis, which can lead to cardiac arrhythmias causing premature sudden cardiac death. FHC is the leading cause of sudden death in the young, most notably young competitive athletes [7, 8]. Generally, FHC is inherited as an autosomal dominant disorder and is genetically heterogeneous. All the FHC mutations identified thus far are in genes encoding the contractile proteins of the cardiac sarcomere including the $\beta$-myosin heavy chain (MyHC) and associated essential and regulatory myosin light chains, and the cardiac protein isoforms of TnT, TnI, actin, and myosin binding protein $\mathrm{C}$, 
and the TPM1 gene encoding $\alpha$-Tm $[6,9]$. Thus, FHC has been termed a "disease of the sarcomere" [5]. There have been four mutations identified in $\alpha$-Tm (TPM1 gene) associated with hypertrophic cardiomyopathy and they account for about 5\% of all cases of FHC (although Tm mutations are more prevalent in the Finnish population, $>25 \%$ ) $[10,11,12,13]$.

In concordance with the varied effects of several other FHC alleles even within given kindreds [14], the FHC mutations in Tm, A63V, K70T, D175N and E180G, are clinically heterogeneous. The D175N mutation has now been identified in several unrelated kindreds, suggesting that this site may be a mutational "hot spot" for the disease [15]. Patients from families with the D175N mutation show varied amounts of cardiac hypertrophy but consistently show a benign clinical outcome with no increased incidence of sudden death. Although the analysis of the A63V, K70T and E180G mutations are limited to small numbers of kindreds, all of these alleles show family histories of sudden cardiac death $[10,11,12,16]$. It is not known why different FHC mutations in the same protein show markedly different disease outcomes. The most attractive hypothesis is that the degree to which each of these mutations changes the ability of Tm to regulate muscle contraction in cardiac muscle might underlie the severity of prognosis of different FHC mutations in $\mathrm{Tm}$.

The position of Tm mutations associated with FHC in the predicted structure of Tm are shown in Fig. 1B. Two of the mutations, E180G and D175N, lie in regions associated with TnT binding [17], which suggests that these mutations might alter the interactions of Tm with the troponin complex. In contrast, the A63V and K70T mutations lie in a region not known to interact with TnT, which suggests these latter two mutations could alter Tm function by different mechanisms. However, in terms of the interactions within the Tm protein itself, the positions of each of the FHC mutations are all highly similar. That is, each of the mutations reside in "e" or "g" positions of the alpha-helical heptad repeat that exists along the entire length of $\mathrm{Tm}$ proteins (Fig. 1C). These residues are generally charged amino acids that are thought to be involved in stabilizing the coiled-coil structure of $\alpha$-Tm [18] and three of the mutations remove charge from those positions. Therefore, all four FHC mutations may destabilize the coiled-coil structure, increase $\mathrm{Tm}$ flexibility in the regions surrounding the mutations and have convergent effects on the ability of $\mathrm{Tm}$ to regulate muscle contraction. In support of this hypothesis, biochemical studies on D175N or E180G mutant tropomyosin homodimers show increased local flexibility about the mutated residues and altered actin binding conformation in the state that allows myosin binding [19].

Another interesting feature of Tm-associated FHC is that although the TPM1 gene that is mutated is expressed in both cardiac and skeletal muscle [3,20], patients with FHC mutations in $\alpha$-Tm do not present a noted skeletal myopathy [10]. This is in contrast to FHC patients with mutations in the $\beta$-MyHC, who often develop central core disease in their slow skeletal muscle fibers [21] because both normal and mutant $\beta$-MyHC proteins are expressed in slow-twitch muscle fibers [22]. It has not been determined why FHC mutations in $\alpha$-Tm do not produce an overt skeletal myopathy.

\section{Tropomyosin mutations and diseases of skeletal muscle: nemaline myopathy}

This absence of a skeletal myopathy associated with FHC mutations in $\mathrm{Tm}$ is quite surprising in light of the recent evidence that mutations in the TPM3 gene, which encodes a nearly identical $\alpha$-Tm expressed in slowtwitch skeletal muscle fibers, produce a skeletal myopathy known as nemaline myopathy (NM) [23]. The TPM3 gene is not expressed in cardiac muscle, which is probably why NM patients with this mutation do not have any evidence of a cardiac myopathy. The TPM3 gene produces a Tm that is nearly identical (>90\%) to TPM1 Tm (Fig. 1A). The methionine that is mutated in TPM3 (M9R) and the entire $\mathrm{N}$-terminal region of Tm are highly conserved among many muscle Tm isoforms from humans to C. elegans (Fig. 1A).

$\mathrm{NM}$ is genetically heterogeneous with autosomal dominant and recessive inheritance; it is also clinically heterogeneous, with neonatal-, childhood-, and adult-onset forms with the most dramatic NM disease phenotype being the "floppy infant" [24]. The hallmarks of NM are severe muscle weakness and structures known as nemaline rods, which are seen within biopsy samples of muscle fibers. Nemaline rods are usually electron-dense enlargements of the muscle Z-line. The origin of the nemaline rods in muscle fibers is unknown, although they are composed primarily of the Z-line protein alpha-actinin which normally helps anchor the actin filament into the Z-line [25]. NM patients often present muscle weakness before nemaline rods are visible, and sometimes nemaline rods are not visible until a second biopsy [24], suggesting that nemaline rods may be a secondary consequence of disease. In addition to Tm, mutations in another contractile protein, skeletal $\alpha$-actin [26], and a structural protein of the skeletal muscle sarcomere, nebulin [27], have been genetically linked to NM. Like TPM3, these gene products are not expressed in adult cardiac muscle either, and this probably explains why most NM patients do not show evidence of the NM phenotype in cardiac muscle. In addition to the M9R mutation in $\alpha$ Tm, which results in an autosomal dominant childhoodonset form of NM, a nonsense mutation at the 31st codon of TPM3 was very recently shown to be associated with a severe neonatal-onset, recessive form of NM [28]. TPM1 is still considered a candidate gene for NM kindred for which mutations have yet to be identified [29], and there is a subset of NM patients who have features of NM, including nemaline rods, present in cardiac muscle [30].

The M9R mutation in $\alpha$-Tm would be predicted to alter the coiled-coil structure of the $\mathrm{N}$-terminus of $\alpha$-Tm. 
The M9R mutation resides in an "a" position (Fig. 1C) of the alpha-helical coiled-coil structure of $\alpha$-Tm which is a "forbidden" position for charged amino acids [31]. The $\mathrm{C}$ - and $\mathrm{N}$-terminus of $\alpha$-Tm are unresolved in the crystal structure of Tm [32], but recent NMR analysis of an $\alpha$-Tm N-terminus peptide suggests that $\alpha$-Tm forms a coiled-coil to the first amino acid on the N-terminus [33]. The N-terminus of $\alpha-T m$, where M9R resides, binds TnT and is also involved in the overlap of adjacent Tm proteins [17]. Biochemical studies have shown that deleting the first nine amino acids or peptidase removal of the last 9-11 amino acids results in $\alpha$-Tm proteins that have negligible or reduced affinity for actin, indicating that the $\mathrm{N}$-terminus of $\mathrm{Tm}$ and the overlap region may contain an important actin binding site $[34,35]$. Therefore, it remains unknown whether the NM mutant Tm, which has an M9R mutation predicted to disrupt the coiled-coil of the $\mathrm{N}$-terminus, is able to assemble normally into the thin filaments of muscle cells.

\section{Tropomyosin mutations in FHC and NM: possible mechanisms of disease pathogenesis}

In general, because the described point mutations in Tm associated with FHC and NM are both inherited as autosomal dominant disorders (patients are heterozygous for the mutant Tm alleles), two general hypotheses of how these mutations might result in FHC and NM have been put forth. The first hypothesis is that the mutations behave as "nulls", meaning that the stable protein is either not expressed or cannot incorporate into the contractile apparatus; therefore, NM and FHC patients suffer from haplo-insufficiency or a reduced amount of Tm protein. The alternative hypothesis is that these mutant proteins incorporate normally into the contractile apparatus and behave as "dominant negatives" by directly altering either the structure or the contractile function of muscle cells.

Most of the evidence that FHC and NM Tm proteins do not behave as "nulls" obtained so far has been indirect. The murine striated muscle $\alpha$-Tm gene has been knocked out by two independent groups [36, 37]. Although the homozygous knock-out (KO) is lethal, the heterozygotes are viable and their cardiac muscle structure and function are indistinguishable from normal. There was no detailed examination of skeletal muscle by either group but there was no gross evidence of a skeletal myopathy. Interestingly, although there is a reduction in the amount of $\alpha$-Tm mRNA in the hearts and skeletal muscle of heterozygous KO mice, the total amount of Tm protein remains unchanged. This suggests that there is post-transcriptional mechanism for maintaining total Tm stoichiometry in the face of reduced amounts of Tm gene transcription [37]. Similarly, when particular contractile proteins, including Tm isoforms, are overexpressed in heart muscle using an $\alpha$-MyHC promoter, the total amount of contractile protein generally remains unchanged $[38,39]$. However, heterozygous murine KO's of $\alpha-\mathrm{MyHC}$ have impaired cardiovascular function, suggesting that "null" alleles of some contractile proteins can affect muscle function [40]. In addition, it is not known whether the FHC or NM mutant Tm proteins are translated but not incorporated into the contractile apparatus, and whether this would affect the stoichiometry of the normal Tm protein.

Both FHC and NM patients show alterations in their sarcomere structure and muscle morphology. Because the contractile proteins are also important structural proteins of the sarcomere, it is not known whether these effects on sarcomeric structure are directly due to the effect of the mutant contractile protein, or are a secondary effect due to compensation for some direct effect of the protein on function. Experiments using the expression of mutant proteins in cultured muscle cells have attempted to elucidate the direct effects of FHC mutant proteins on the sarcomeric structure in the absence of compensatory adaptations that might occur in vivo. The results from these experiments are disparate. The expression of FHC mutant $\beta$-MyHC in neonatal cardiac myocytes results in incorporation of the protein without disrupting sarcomere structure [41], while the expression of the same mutant protein in feline adult cardiac myocytes by recombinant adenovirus disrupts the cardiac sarcomere in a portion of the cells (although incorporation of this mutant protein was not demonstrated in the latter experiments) [42]. Expression of FHC mutant TnT proteins has been shown to have no direct effect on sarcomere structure in rat and feline adult cardiac myocytes [43, 44], while different mutations in TnT disrupt sarcomere structure in a small percentage of cells when the proteins are expressed at high levels in quail myotubes [45]. Until recently, no experiments had examined whether expression of FHC or NM mutant $\alpha$-Tm directly affects sarcomere structure. However, nemaline rods can be found in a variety of conditions or myopathies unrelated to NM, including AIDS infection, hemodialysis, and central core disease [24]. The finding that muscle weakness can precede the development of nemaline rods suggests the sarcomere alterations may be a secondary, and potentially non-specific, consequence of NM [24]. Clearly, experiments examining the direct effects of expression of these mutant $\alpha$-Tm proteins on sarcomeric structure in muscle cells in vitro would have important implications for the understanding of FHC and NM pathogenesis.

Gene transfer and muscle transgenesis: genetic systems to understand the function of mutant Tm proteins

The present time is an exciting stage in muscle research because of the recent development of experimental tools that allow one to genetically manipulate cardiac muscle cells both in vitro and in vivo $[39,46]$. Both approaches, namely transgenic animals and gene transfer to isolated adult cardiac myocytes, have the capability of advancing our understanding of the role of contractile protein mutations in the pathogenesis of disease. Several laboratories 
have used the $\alpha$-MyHC promoter for expression and replacement of contractile proteins in adult mouse heart muscle in vivo [47, 48, 49, 50, 51, 52, 53]. In addition, investigators have used gene targeting to create knock-in FHC mutations for MyHC and MyBP-C [54, 55]. Many of these animal models show morphological hallmarks of FHC, including myocyte disarray and interstitial fibrosis, but variable effects on cardiac hypertrophy. Clearly, animal models of disease are critical for understanding how these mutant proteins might alter organ function, and perhaps for understanding the secondary phenomena that occur in these diseases. However, animal models may be difficult to interpret because any phenotype measured, morphological or functional, could be due to either the direct effect caused by that protein and/or the compensatory adaptations the animal has employed to counteract an initial defect. Therefore, in order to sort out what are the primary defects in muscle cells caused by these mutant proteins with the goal of understanding the primary mechanisms of disease pathogenesis, an alternative approach would provide important additional insight.

A complementary approach to identifying the direct effects of mutant contractile proteins on the structure and function of muscle cells is to use gene transfer into isolated, fully differentiated adult cardiac myocytes. Using isolated cells allows the assessment of the direct effects of mutant proteins on the structure and function of muscle cells without the compensatory adaptations that might occur in vivo to maintain homeostasis and the life of the organism. Adenoviral vectors provide a highly efficient method of rapidly modifying fully differentiated adult cardiac myocytes in vitro without altering the morphological stability, contractile protein isoform expression and stoichiometry, or isometric force production of the muscle cells [56]. Westfall et al. [57] demonstrated that adenoviral gene transfer to adult cardiac myocytes combined with single cell isometric force measurements provides a new method for studying the structure-function relationship of a contractile protein $(\mathrm{TnI})$ in the context of a fully differentiated adult muscle cell [57]. Adenoviral gene transfer to adult cardiac myocytes has been used previously to study the effect of FHC mutations in $\beta$-MyHC on the structure of adult cardiac myocytes [42] and the effects of FHC mutations in TnT on the structure and contractile function of adult cardiac myocytes $[43,44]$.

Thus far, other methods for studying mutant contractile proteins in fully functional muscle cells have been not readily applicable to the study of Tm structure/function, probably because of the integral role of $\mathrm{Tm}$ in maintaining the structure of the thin filament. Extraction and reconstitution have been used by several investigators to study TnI and TnT structure/function and the potential dominant negative effects of FHC mutations in permeabilized muscle fibers (for review see Redwood et al. [58]). But even for these more readily extracted proteins, perturbations in force production are often seen even after reconstituting wild-type (WT) TnT or TnI pro- teins, thus the quality and quantity of extraction/ reconstitution when trying to compare mutant contractile proteins can potentially affect the interpretation of these results. Fujita et al. [59] have reported methods for reconstituting entire thin filaments including actin, Tm and Tn into gelsolin-treated (actin-extracted) cardiac fibers. However, large variations in force recovery occur following reconstitution with actin (30-300\%), and further deterioration of force occurs after addition of $\mathrm{Tm}$ and Tn, suggesting that several unexplained, non-specific effects of reconstitution are occurring. In addition, cultured, differentiating quail myotubes, used previously to study the functional effects of FHC TnT, do not tolerate the transfection, selection and overexpression of normal Tm [60, 61]. Finally, Redwood et al. [62] has recently shown that thin filaments reconstituted with $50 \%$ or $100 \%$ FHC mutant TnT behave in an opposite manner in the in vitro motility assay, suggesting that the percentage of mutant protein may be an important factor when evaluating the relevance of the findings of these assays to patients heterozygous for FHC mutations.

In this review, we describe recent experiments in which gene transfer to adult cardiac myocytes has been developed as a model system and used to understand and compare how FHC and NM mutations in Tm directly alter the structure and function of striated muscle cells to gain an insight into the primary mechanisms of FHC and NM pathogenesis. Gene transfer provides a unique insight into the direct effects that these myopathy-causing Tm mutations have on the structure and function of fully differentiated striated muscle cells. These findings should have important implications not only for understanding the primary mechanisms of important human diseases but also for understanding the role of $\mathrm{Tm}$ in the regulation of normal striated muscle contraction.

\section{Gene transfer of Tm to adult cardiac myocytes: direct effects of FHC and NM mutations on $\mathrm{Tm}$ regulation of muscle contraction}

In order to create a stable muscle system in which to study the structure and function of genetically modified contractile proteins, protocols have been developed to maintain stable, fully differentiated adult cardiac myocytes in primary culture in conjunction with extremely efficient adenoviral gene transfer $(>90 \%$ of the myocytes) $[46,56]$. Adenoviral-mediated gene transfer was shown to be an effective and efficient method for replacing a portion (approaching, on average, $35-45 \%$ of total $\mathrm{Tm}$ in the muscle cell) of the endogenous $\alpha-\mathrm{Tm}$ protein in adult rat cardiac myocytes with human $\alpha-\operatorname{Tm}$ [63]. In addition, the use of epitope tagging, quantitative Western blotting and immunofluorescence confocal microscopy allowed the direct visualization and incorporation of newly synthesized $\alpha-\operatorname{Tm}$ and cTnI into the fully differentiated muscle sarcomere. These experiments identified how important contractile proteins are replaced within the thin filaments of fully differentiated muscle contrac- 
tile apparatus [63]. Interestingly, the amount of expression and incorporation of $\mathrm{Tm}$ and $\mathrm{cTnI}$ achieved by gene transfer over time in culture appears to be limited by the turnover of the protein already residing in the myofilaments [63]. The initial incorporation of $\alpha$-Tm into the region about the pointed end of the actin thin filament, and random incorporation of $\mathrm{TnI}$ into the fully differentiated sarcomere suggest a model of thin filament maintenance, in which actin thin filaments remain for the most part intact, with the mechanism of removal and replacement of contractile proteins with newly synthesized proteins being highly dependent on the structural properties and molecular interactions of the protein in the sarcomere. Most importantly, the expression of human Tm by gene transfer did not change total Tm stoichiometry or the $\mathrm{Ca}^{2+}$-activated isometric force production of the cardiac myocytes [63], which demonstrates that specific replacement of a portion of the Tm in adult rat cardiac myocytes with WT human $\alpha$-Tm can be achieved without altering the contractile function of a fully differentiated muscle cell.

In following studies, the gene transfer of normal and mutant human $\alpha$-Tm to adult cardiac myocytes was used to determine the effects of FHC and NM mutations on the ability of $\mathrm{Tm}$ to incorporate into the muscle sarcomere and, ultimately, on the ability of Tm to regulate contraction in response to changing $\mathrm{Ca}^{2+}$ concentrations. The analogous NM mutation in TPM3 was made in the nearly identical TPM1 $\alpha$-Tm (M8R, see Fig. 1A) for direct comparison of this NM mutant Tm to the FHC mutations in Tm. Interestingly, using epitope tagging and immunofluorescence confocal microscopy to directly follow the incorporation of the newly expressed $\mathrm{Tm}$ in adult cardiac myocytes, all four FHC mutant $\alpha$-Tm and the NM mutant $\alpha$-Tm were shown to incorporate into sarcomeres similar to WT $\alpha-\operatorname{Tm}[64,65]$. Quantitative Western blotting showed that the expression level achieved with all four FHC mutants and NM mutants was similar to WT $\alpha$-Tm $(\cong 40 \%$ total Tm) without altering total Tm stoichiometry $[64,65]$. Given that the FHC and NM mutants can be expressed and incorporated into sarcomeres similar to WT Tm (and in some experiments were shown to be able to directly compete with the endogenous Tm; see [65] for details), the partial replacement of $\mathrm{Tm}$ by the mutant $\mathrm{Tm}$ is indeed desirable, because FHC and NM patients are heterozygous for each of the mutant alleles. The gross sarcomeric structural alterations evident in FHC and NM patients were not seen in muscle cells expressing FHC or NM mutant Tm 5-6 days after the gene transfer. These results suggest that the FHC and NM mutant Tm proteins do not directly alter sarcomeric structure and that these alterations in sarcomeric structure are secondary and long-term consequences of disease pathogenesis.

Most strikingly, expression of the FHC and NM mutant $\alpha$-Tm proteins had differential effects on the $\mathrm{Ca}^{2+}$ sensitivity of force production which may be related to the different clinical presentation of these mutations (NM mutations produce a skeletal muscle myopathy,

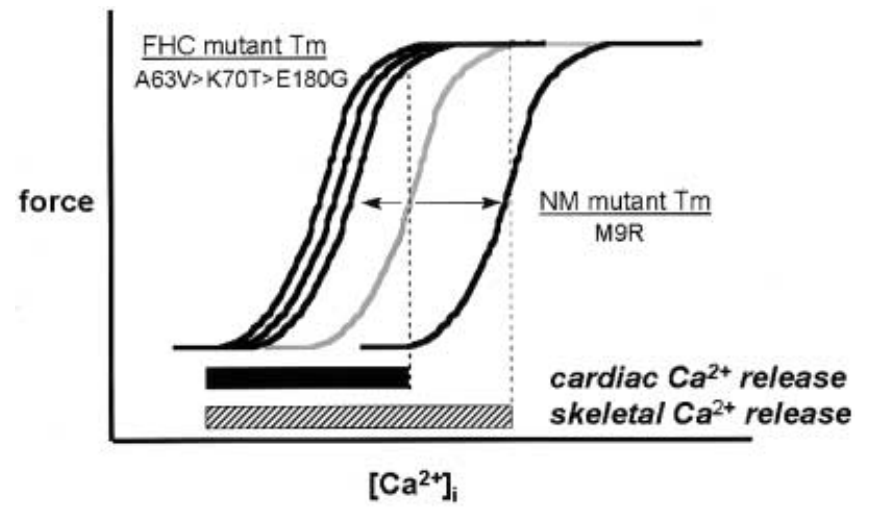

Fig. 2 Schematic summary of the effect of hypertrophic cardiomyopathy $(F H C)$ and nemaline myopathy $(N M)$ mutations in $\alpha-$ $\mathrm{Tm}$ on $\mathrm{Ca}^{2+}$-activated force generation in fully differentiated muscle cells $[64,65]$. The trace in gray represents a muscle cell expressing wild-type $\alpha$-Tm. The black lines indicate the representative shifts in $\mathrm{Ca}^{2+}$ sensitivity in muscle cells expressing either HCM or NM mutant Tm. Shown by the bars on the bottom is the range of $\mathrm{Ca}^{2+}$ release during a normal cardiac muscle (solid bar) or skeletal muscle (hatched bar) contraction [66]. As shown, $\mathrm{Ca}^{2+}$ released during normal cardiac contraction does not maximally activate cardiac muscle. However, in skeletal muscle tetanic contractions, enough $\mathrm{Ca}^{2+}$ is released to nearly fully activate skeletal muscle contraction [68]

FHC mutations do not). Neither FHC nor NM mutant Tm expression altered the maximal force production of the fully differentiated muscle cells [64, 65]. However, the FHC mutations increased the $\mathrm{Ca}^{2+}$ sensitivity of contraction in $\alpha-\mathrm{Tm}$, with the following allele hierarchy: $\mathrm{A} 63 \mathrm{~V}>\mathrm{K} 70 \mathrm{~T}>\mathrm{E} 180 \mathrm{G}>>\mathrm{D} 175 \mathrm{~N} \cong \mathrm{WT}$ [65]. In contrast, the NM mutant $\alpha$-Tm caused a decrease in $\mathrm{Ca}^{2+}$ sensitivity (Fig. 2) [64]. These results suggest the FHC and NM mutations differentially modulate the ability of $\mathrm{Tm}$ to regulate muscle contraction in response to changing $\mathrm{Ca}^{2+}$ concentrations. These data provide evidence that defects in the ability of $\alpha-T m$ to regulate contractile function may be an important determinant in the differential mechanisms that underlie the pathogenesis of mutant Tm-associated FHC and NM.

\section{FHC $\alpha$-Tm: $\mathrm{Ca}^{2+}$ hypersensitivity and disease pathogenesis}

The gene transfer experiments identified a direct, convergent effect of three of the FHC $\alpha$-Tm mutations, $\mathrm{A} 63 \mathrm{~V}, \mathrm{~K} 70 \mathrm{~T}$, and E180G, namely to increase the $\mathrm{Ca}^{2+}$ sensitivity of force production. D175N $\alpha$-Tm did not alter $\mathrm{Ca}^{2+}$ sensitivity of contraction at the levels of expression seen in these experiments. It cannot be ruled out that $\mathrm{D} 175 \mathrm{~N} \alpha-\mathrm{Tm}$ has a primary effect on some other aspect of contraction not measured in these studies. However, recent evidence from a transgenic mouse model suggests that higher levels of expression ( $>60 \%$ of total $\mathrm{Tm})$ of $\mathrm{D} 175 \mathrm{~N} \alpha-\mathrm{Tm}$ in mouse heart can increase the $\mathrm{Ca}^{2+}$ sensitivity of force production in isolated cardiac muscle strips [53], while lines that had levels of expres- 
sion similar to those seen in the gene transfer experiments $\left(40 \%\right.$ of total $\mathrm{Tm}$ ) had no alterations in $\mathrm{Ca}^{2+}$ sensitivity. These experiments suggest that the effects of FHC mutations in $\alpha$-Tm on $\mathrm{Ca}^{2+}$-activated force production are probably all convergent, but that the threshold expression for D175N $\alpha$-Tm to have an effect on $\mathrm{Ca}^{2+}$ sensitivity appears to be higher than that of the other three mutations, which is consistent with the benign presentation of the D175N allele [15]. Thus, the hierarchy of the effect of each Tm allele to alter contractile function appears to be related to the severity of the clinical presentation of each allele. This convergent hypersensitivity of $\mathrm{Ca}^{2+}$-activated force production might be expected to have large effects on force production in cardiac muscle (Fig. 2) because normal $\mathrm{Ca}^{2+}$ release in cardiac muscle only reaches submaximal activation levels [66]. In addition, as $\mathrm{Ca}^{2+}$ is sequestered within the cardiac myocyte, it is possible that force would remain high and relaxation of the cardiac myocyte might be slowed, contributing to the diastolic dysfunction and reduced chamber filling seen in FHC patients [67].

The effect of FHC mutations to increase $\mathrm{Ca}^{2+}$ sensitivity without affecting maximal force production may also explain why FHC patients with Tm mutations do not show a skeletal muscle myopathy. The $\mathrm{Ca}^{2+}$ released during a skeletal muscle contraction (especially tetanic contractions) reaches concentrations that maximally activate skeletal muscle (Fig. 2) [68]. Therefore, FHC mutant Tm would probably not augment the maximal force production during normal $\mathrm{Ca}^{2+}$ release in skeletal muscle, and, therefore, not provide a stimulus for remodeling in skeletal muscle that results in myopathy. The lack of muscle cell disarray in skeletal muscle expressing FHC mutant Tm also further strengthens the conclusion, from the gene transfer studies, that sarcomere disarray caused by FHC mutant Tm is a secondary consequence of functional changes that are specific to the myocardium.

NM mutant $\alpha$-Tm: $\mathrm{Ca}^{2+}$ hyposensitivity and disease pathogenesis

Gene transfer experiments have shown that the NM mutation in $\alpha$-Tm (M9R) exerts a "dominant negative" effect that decreases the $\mathrm{Ca}^{2+}$ sensitivity of force production [64]. This finding supports the hypothesis that differential effects of FHC and NM mutations on the ability of $\alpha-T m$ to regulate muscle contraction underlie the differential clinical presentations of NM and FHC. Clearly, NM mutations in Tm do not produce a cardiac myopathy because the TPM3 gene that is mutated is not expressed in cardiac muscle. Unlike the FHC mutations in Tm expressed in skeletal muscle, which do not produce a skeletal muscle myopathy, the NM mutations in Tm produce a noted skeletal muscle myopathy characterized by skeletal muscle weakness. In that regard, it is noteworthy that expression of the NM mutant Tm decreases the $\mathrm{Ca}^{2+}$ sensitivity of force production. Thus, in skeletal muscle where the released $\mathrm{Ca}^{2+}$ nearly maximally activates the

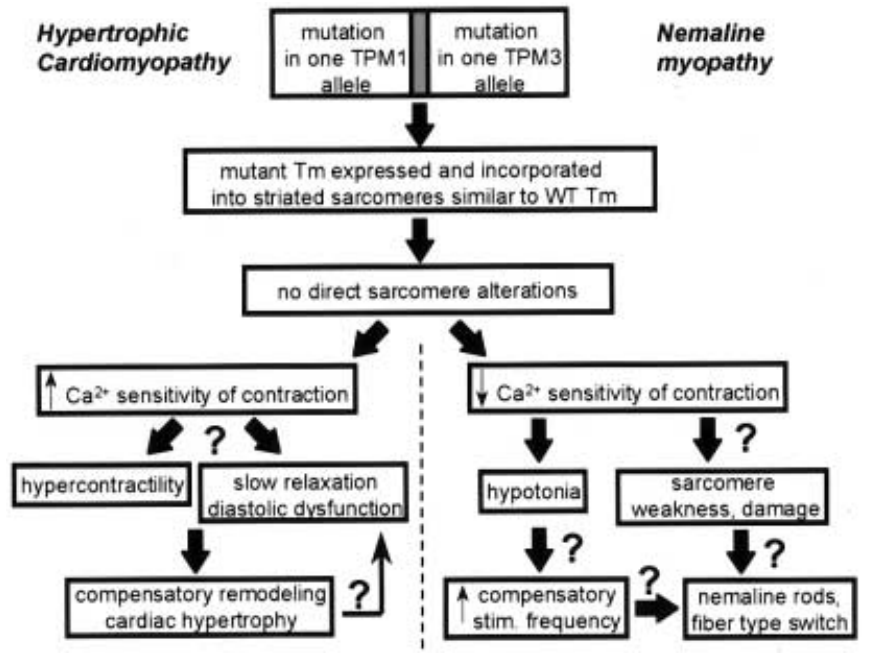

Fig. 3 Summary of the differential direct effects of FHC and NM mutant $\mathrm{Tm}$ on the regulation of muscle contraction and the relevance to the clinical presentation of FHC and NM

contractile apparatus, the NM mutant Tm may reduce force production during the normal release of $\mathrm{Ca}^{2+}$, and this may in part explain the muscle weakness seen in NM (Fig. 2).

In the gene transfer experiments nemaline rod formation did not occur directly as a response to NM mutant $\alpha$-Tm expression in quiescent myocytes or in myocytes undergoing unloaded contraction [63]. Several arguments favor the interpretation that NM rods are perhaps not a direct effect of NM mutant $\alpha$-Tm expression. First, some NM patients do have nemaline rods within their cardiac muscle, suggesting that nemaline rods can form in heart muscle cells (if the mutant genes are expressed there) [30]. Thus, the isolated heart cell seems to be an appropriate model system of striated muscle for studying NM rod formation. Second, as mentioned before, NM rods appear to be a secondary consequence of multiple changes in muscle physiology, such as HIV infection [69], and thus may not be a specific, direct consequence of a mutant sarcomere protein [24].

The mechanism behind the formation of the nemaline rods is unknown, because nemaline rod formation was not seen in fully differentiated striated muscle cells in vitro expressing NM mutant $\alpha$-Tm. If the distribution of NM $\alpha-T m$ is not uniform within a given muscle fiber, NM $\alpha$-Tm-expressing sarcomeres may not be able to produce as much force as other sarcomeres at similar $\mathrm{Ca}^{2+}$ concentration (Fig. 3). This heterogeneity of the ability of sarcomeres to bear force has been hypothesized to be directly involved in certain regions of muscle being more susceptible to contraction-induced injury [70]. Similarly, when muscles contract against no load (as shown in tenotomized muscles of rat) nemaline rods can also form [71]. In this tenotomized rat experimental model, the rod formation is dependent on the muscle being innervated. Thus, there may be interplay between the load on the sarcomeres and the compensatory adaptations that are trying to maintain force production in the 
muscle that lead to nemaline rod formation in vivo. NM patients with the M9R mutation have a type II fiber predominance, which is indirect evidence that changes in muscle stimulus frequency may be occurring as a compensatory change in NM patients [23]. If long-term cell cultures of fully differentiated striated muscle cells could be developed, it may be possible to test the hypothesis that nemaline rod formation is load dependent by culturing muscle cells on elastic membranes [72] and imposing length changes to contracting myocytes expressing NM mutant $\alpha$-Tm.

\section{FHC and NM $\alpha$-Tm: tools to tease out the role of $\alpha-\mathrm{Tm}$ in contraction}

The finding that FHC and NM mutant $\alpha$-Tm differentially alter the $\mathrm{Ca}^{2+}$ regulation of steady-state isometric force production in permeabilized muscle cells was both exciting and fortuitous, because these mutations provided insight into the role of $\alpha$-Tm in the regulation of muscle contraction. McKillop and Geeves [73] have proposed a three-state model of thin filament regulation that features a blocked state that does not allow myosin binding, a closed state that allows weak myosin binding, and an open state that is induced by myosin binding and allows strong, force-producing myosin binding to occur. The three states are proposed to exist in equilibrium, with a large proportion of the thin filaments in the blocked state and a small portion in the closed state in the absence of $\mathrm{Ca}^{2+}$ [73]. In this model, the major $\mathrm{Ca}^{2+}$ sensitive step is a shift in the equilibrium from blocked to closed such that the closed state is more heavily populated. It might seem likely that the FHC mutations in $\alpha$ $\mathrm{Tm}$ affect this step because the $\mathrm{Ca}^{2+}$ sensitivity of contraction increased. However, if this step is altered such that the closed state is more highly populated in the absence of $\mathrm{Ca}^{2+}$, than an increase in force in the absence of $\mathrm{Ca}^{2+}$ might be expected because myosin heads could bind and induce an open state. No increase in force in the absence of $\mathrm{Ca}^{2+}$ was seen with FHC mutant Tm expression in adult cardiac myocytes (D.M. and J.M., unpublished data). Thus, it could be hypothesized that the conformational changes caused by the FHC mutations in $\alpha-T m$ shift the equilibrium between closed and open to the open state. Thus, at any given $\mathrm{Ca}^{2+}$ concentration, the likelihood of myosin attaching and undergoing a transition to a force-producing state is increased, resulting in an increase in the $\mathrm{Ca}^{2+}$ sensitivity of contraction. The situation for the NM mutations on the stability of various thin filaments states is less clear. Clearly, a large percentage of the blocked state is required for full inhibition and relaxation. But force measurements really only examine the open state (where crossbridge cycling and force production can occur). Thus, NM mutations in $\alpha$-Tm could be inhibiting the blocked to closed transition or the closed to open transition.

The convergent phenotype of the FHC mutations, and their convergent position in the coiled-coil structure of
$\alpha-\mathrm{Tm}$, indicates that important conformational changes in $\alpha$-Tm may be occurring in the regions around amino acids 63-70 and 175-180 during the transition from closed to open states. These regions may need to be flexible for $\alpha$-Tm to adopt an open conformation, and thus increasing the flexibility about these regions stabilizes these states. Alternatively, these residues could be involved in electrostatic interactions between $\alpha-\mathrm{Tm}$ and the actin filament. Molecular modeling studies of thin filament reconstructions have shown that the interaction of $\alpha-\mathrm{Tm}$ and the actin filament might be primarily electrostatic [74], so perhaps the residues that remove charge are causing a local charge change that stabilizes the open state. However, the A63V mutation produced the largest change in the $\mathrm{Ca}^{2+}$ sensitivity of contraction and this residue change does not effect the electrostatic charge. In contrast to the FHC mutations, the destabilization of the coiled coil in the N-terminus of $\alpha-\mathrm{Tm}$ by the M9R NM mutation may alter the interactions between adjacent $\alpha$ Tm proteins or perhaps TnT. Disruption of the N-terminus by deletion or exon swapping has been shown to block or alter $\alpha-\mathrm{Tm}$ binding to actin in the absence of Tn [34]. Because $\alpha-T m$, in the absence of $\mathrm{Tn}$, has been shown to bind to muscle actin in different states depending on the tropomyosin isoform, Tm appears to move fairly easily between different states [75]. Perhaps disruption of the coiled-coil near the N-terminus destabilizes the closed conformation enough to reduce the equilibrium constant for the transition from blocked to closed which would reduce the $\mathrm{Ca}^{2+}$ sensitivity of force production.

\section{Future directions}

The results from the comparative gene transfer studies have shown that the FHC and NM mutations differentially alter the steady-state $\mathrm{Ca}^{2+}$-activated force production. But cardiac myocytes normally respond to transient changes in intracellular $\mathrm{Ca}^{2+}$ concentration. Therefore, an important next step will be to determine if and how FHC and NM mutant Tm proteins differentially alter intact muscle cell contraction. Further comparison of the effects of FHC and NM mutations on the biomechanical properties of intact muscle cells and exploring strong crossbridges as activating ligands in permeabilized muscle cells [76] will provide important insights into the role of $\mathrm{Tm}$ in the regulation of myocyte contraction under physiological and pathophysiological conditions.

Extrapolating the results of studies of isolated cardiac myocytes to the function of the whole organ is an important consideration and, therefore, animal models of FHC and NM need to be examined. Recently, Muthuchamy et al. [53] described a transgenic mouse line expressing FHC mutant D175N murine $\alpha$-Tm in the adult heart using an $\alpha$-MyHC promoter. The phenotype of these mice was remarkably mild. Transgenic mice showed no gross ventricular hypertrophy, small systolic and diastolic function abnormalities and only mild disruption of the 
myocyte structure in less than $5 \%$ of the myocardium. The results from the gene transfer experiments suggest that the other three mutations in $\alpha$-Tm might produce a more profound phenotype in transgenic animals. It would also be interesting to compare the effects of expression of each of the FHC mutant Tm on the contractile function of isolated cardiac myocytes from transgenic animals with the effects of FHC mutants on contraction by gene transfer to identify potential in vivo compensatory adaptations and how they contribute to additional alterations in the mechanical function of individual muscle cells.

The mutations in $\alpha$-Tm associated with FHC and NM appear to directly alter the regulatory behavior of the thin filament in response to changes in intracellular $\left[\mathrm{Ca}^{2+}\right]$. Therefore, based on the in vitro experiments it could be proposed that gene transfer experiments overexpressing normal $\alpha$-Tm should be able to eventually replace the mutant $\alpha$-Tm in FHC- or NM-expressing muscle cells and potentially correct FHC or NM. Although the gene therapy field is still in its infancy, future experiments showing the feasibility of this approach to animal models of FHC or NM both in vitro and in vivo will be important first steps. Alternatively, pharmacological intervention targeted at modifying the $\mathrm{Ca}^{2+}$ transient or the thin filament response to intracellular $\mathrm{Ca}^{2+}$ may be able to correct or prevent symptoms of FHC or NM. For instance, a class of drugs known as $\mathrm{Ca}^{2+}$ sensitizers were originally developed for the treatment of heart failure [77]. Many of these drugs directly increase the affinity of $\mathrm{Ca}^{2+}$ binding to troponin $\mathrm{C}$ (although these drugs are also potent phosphodiesterase inhibitors). Perhaps new classes of $\mathrm{Ca}^{2+}$ sensitizers that target the thin filament regulatory proteins, including $\mathrm{Tm}$, may be therapeutic tools for treating NM. Conversely, $\mathrm{Ca}^{2+}$ channel antagonists, such as verapamil and diltiazem, which decrease $\mathrm{Ca}^{2+}$ release may be therapeutic and potentially preventative for FHC. Clinical studies suggest that verapamil and diltiazem can improve ventricular filling in hypertrophic cardiomyopathy $[67,78]$. Consequently, studies of the direct effects of mutant contractile proteins associated with cardiac and skeletal myopathies on the structure and function of muscle cells may point to important new targets and tools for the therapy and prevention of disease pathogenesis.

\section{References}

1. Tobacman LS (1996) Thin filament-mediated regulation of cardiac contraction. Annu Rev Physiol 58:447-481

2. Gordon AM, Homsher E, Regnier M (2000) Regulation of contraction in striated muscle. Physiol Rev 80:853-924

3. Lees-Miller JP, Helfman DM (1991) The molecular basis for tropomyosin isoform diversity. Bioessays 13:429-437

4. Schiaffino S, Reggiani C (1996) Molecular diversity of myofibrillar proteins: gene regulation and functional significance. Physiol Rev 76:371-423

5. Watkins H, Seidman JG, Seidman CE (1995) Familial hypertrophic cardiomyopathy: a genetic model of cardiac hypertrophy. Hum Mol Genet 4:1721-1727
6. Bonne G, Carrier L, Richard P, Hainque B, Schwartz K (1998) Familial hypertrophic cardiomyopathy: from mutations to functional defects. Circ Res 83:580-593

7. Maron BJ, Casey SA, Poliac LC, Gohman TE, Almquist AK, Aeppli DM (1999) Clinical course of hypertrophic cardiomyopathy in a regional United States cohort [published erratum appears in J Am Med Assoc 1999 Jun 23-30;281(24):2288]. J Am Med Assoc 281:650-655

8. Maron BJ, Shirani J, Poliac LC, Mathenge R, Roberts WC, Mueller FO (1996) Sudden death in young competitive athletes. Clinical, demographic, and pathological profiles. J Am Med Assoc 276:199-204

9. Mogensen J, Klausen IC, Pedersen AK, Egeblad H, Bross P, Kruse TA, Gregersen N, Hansen PS, Baandrup U, Borglum AD (1999) Alpha-cardiac actin is a novel disease gene in familial hypertrophic cardiomyopathy. J Clin Invest 103:R39R43

10. Thierfelder L, Watkins H, MacRae C, Lamas R, McKenna W, Vosberg HP, Seidman JG, Seidman CE (1994) Alpha-tropomyosin and cardiac troponin T mutations cause familial hypertrophic cardiomyopathy: a disease of the sarcomere. Cell 77: 701-712

11. Nakajima-Taniguchi $\mathrm{C}$, Matsui $\mathrm{H}$, Nagata $\mathrm{S}$, Kishimoto $\mathrm{T}$, Yamauchi-Takihara K (1995) Novel missense mutation in alpha-tropomyosin gene found in Japanese patients with hypertrophic cardiomyopathy. J Mol Cell Cardiol 27:2053-2058

12. Yamauchi-Takihara $K$, Nakajima-Taniguchi $C$, Matsui $H$, Fujio Y, Kunisada K, Nagata S, Kishimoto T (1996) Clinical implications of hypertrophic cardiomyopathy associated with mutations in the alpha-tropomyosin gene. Heart 76:63-65

13. Jaaskelainen P, Soranta M, Miettinen R, Saarinen L, Pihlajamaki J, Silvennoinen K, Tikanoja T, Laakso M, Kuusisto J (1998) The cardiac beta-myosin heavy chain gene is not the predominant gene for hypertrophic cardiomyopathy in the Finnish population. J Am Coll Cardiol 32:1709-1716

14. Fananapazir L, Epstein ND (1994) Genotype-phenotype correlations in hypertrophic cardiomyopathy. Insights provided by comparisons of kindreds with distinct and identical beta-myosin heavy chain gene mutations. Circulation 89:22-32

15. Coviello DA, Maron BJ, Spirito P, Watkins H, Vosberg HP, Thierfelder L, Schoen FJ, Seidman JG, Seidman CE (1997) Clinical features of hypertrophic cardiomyopathy caused by mutation of a "hot spot" in the alpha-tropomyosin gene. J Am Coll Cardiol 29:635-640

16. Thierfelder L, MacRae C, Watkins H, Tomfohrde J, Williams M, McKenna W, Bohm K, Noeske G, Schlepper M, Bowcock A (1993) A familial hypertrophic cardiomyopathy locus maps to chromosome 15q2. Proc Natl Acad Sci USA 90:6270-6274

17. White SP, Cohen C, Phillips GN Jr. (1987) Structure of cocrystals of tropomyosin and troponin. Nature 325:826-828

18. McLachlan AD, Stewart M (1975) Tropomyosin coiled-coil interactions: evidence for an unstaggered structure. J Mol Biol 98:293-304

19. Golitsina N, An Y, Greenfield NJ, Thierfelder L, Iizuka K, Seidman JG, Seidman CE, Lehrer SS, Hitchcock-DeGregori SE (1997) Effects of two familial hypertrophic cardiomyopathy-causing mutations on alpha-tropomyosin structure and function [published erratum appears in Biochemistry 1999 Mar 23;38(12):3850]. Biochemistry 36:4637-4642

20. MacLeod AR, Gooding C (1988) Human hTM alpha gene: expression in muscle and nonmuscle tissue. Mol Cell Biol $8: 433-440$

21. Fananapazir L, Dalakas MC, Cyran F, Cohn G, Epstein ND (1993) Missense mutations in the beta-myosin heavy-chain gene cause central core disease in hypertrophic cardiomyopathy. Proc Natl Acad Sci USA 90:3993-3997

22. Cuda G, Fananapazir L, Zhu WS, Sellers JR, Epstein ND (1993) Skeletal muscle expression and abnormal function of beta-myosin in hypertrophic cardiomyopathy. J Clin Invest 91:2861-2865

23. Laing NG, Wilton SD, Akkari PA, Dorosz S, Boundy K, Kneebone C, Blumbergs P, White S, Watkins H, Love DR 
(1995) A mutation in the alpha tropomyosin gene TPM3 associated with autosomal dominant nemaline myopathy [published erratum appears in Nature Genet 1995 Jun;10(2):249]. Nature Genet 9:75-79

24. North KN, Laing NG, Wallgren-Pettersson C (1997) Nemaline myopathy: current concepts. The ENMC International Consortium and Nemaline Myopathy [published erratum appears in J Med Genet 1997 Oct;34(10):879]. J Med Genet 34:705713

25. Jockusch BM, Veldman H, Griffiths GW, van Oost BA, Jennekens FG (1980) Immunofluorescence microscopy of a myopathy. alpha-Actinin is a major constituent of nemaline rods. Exp Cell Res 127:409-420

26. Nowak KJ, Wattanasirichaigoon D, Goebel HH, Wilce M, Pelin K, Donner K, Jacob RL, Hubner C, Oexle K, Anderson JR, Verity CM, North KN, Iannaccone ST, Muller CR, Nurnberg P, Muntoni F, Sewry C, Hughes I, Sutphen R, Lacson AG, Swoboda KJ, Vigneron J, Wallgren-Pettersson C, Beggs AH, Laing NG (1999) Mutations in the skeletal muscle alpha-actin gene in patients with actin myopathy and nemaline myopathy. Nature Genet 23:208-212

27. Pelin K, Hilpela P, Donner K, Sewry C, Akkari PA, Wilton SD, Wattanasirichaigoon D, Bang ML, Centner T, Hanefeld F, Odent S, Fardeau M, Urtizberea JA, Muntoni F, Dubowitz V, Beggs AH, Laing NG, Labeit S, de la CA, Wallgren-Pettersson C (1999) Mutations in the nebulin gene associated with autosomal recessive nemaline myopathy. Proc Natl Acad Sci USA 96:2305-2310

28. Tan P, Briner J, Boltshauser E, Davis MR, Wilton SD, North K, Wallgren-Pettersson C, Laing NG (1999) Homozygosity for a nonsense mutation in the alpha-tropomyosin slow gene TPM3 in a patient with severe infantile nemaline myopathy. Neuromusc Disord 9:573-579

29. Wallgren-Pettersson C, Pelin K, Hilpela P, Donner K, Porfirio B, Graziano C, Swoboda KJ, Fardeau M, Urtizberea JA, Muntoni F, Sewry C, Dubowitz V, Iannaccone S, Minetti C, Pedemonte M, Seri M, Cusano R, Lammens M, CastagnaSloane A, Beggs AH, Laing NG, de la CA (1999) Clinical and genetic heterogeneity in autosomal recessive nemaline myopathy. Neuromusc Disord 9:564-572

30. Ishibashi-Ueda H, Imakita M, Yutani C, Takahashi S, Yazawa K, Kamiya T, Nonaka I (1990) Congenital nemaline myopathy with dilated cardiomyopathy: an autopsy study. Hum Pathol 21:77-82

31. Kohn WD, Mant CT, Hodges RS (1997) Alpha-helical protein assembly motifs. J Biol Chem 272:2583-2586

32. Whitby FG, Phillips GN Jr (2000) Crystal structure of tropomyosin at 7 Angstroms resolution. Proteins 38:49-59

33. Greenfield NJ, Montelione GT, Farid RS, Hitchcock-DeGregori SE (1998) The structure of the N-terminus of striated muscle alpha-tropomyosin in a chimeric peptide: nuclear magnetic resonance structure and circular dichroism studies. Biochemistry 37:7834-7843

34. Cho YJ, Liu J, Hitchcock-DeGregori SE (1990) The amino terminus of muscle tropomyosin is a major determinant for function. J Biol Chem 265:538-545

35. Mak AS, Smillie LB (1981) Non-polymerizable tropomyosin: preparation, some properties and F-actin binding. Biochem Biophys Res Commun 101:208-214

36. Blanchard EM, Iizuka K, Christe M, Conner DA, GeisterferLowrance A, Schoen FJ, Maughan DW, Seidman CE, Seidman JG (1997) Targeted ablation of the murine alpha-tropomyosin gene. Circ Res 81:1005-1010

37. Rethinasamy P, Muthuchamy M, Hewett T, Boivin G, Wolska BM, Evans C, Solaro RJ, Wieczorek DF (1998) Molecular and physiological effects of alpha-tropomyosin ablation in the mouse. Circ Res 82:116-123

38. Muthuchamy M, Grupp IL, Grupp G, O'Toole BA, Kier AB, Boivin GP, Neumann J, Wieczorek DF (1995) Molecular and physiological effects of overexpressing striated muscle betatropomyosin in the adult murine heart. J Biol Chem 270: 30593-30603
39. James J, Robbins J (1997) Molecular remodeling of cardiac contractile function. Am J Physiol 273:H2105-H2118

40. Jones WK, Grupp IL, Doetschman T, Grupp G, Osinska H, Hewett TE, Boivin G, Gulick J, Ng WA, Robbins J (1996) Ablation of the murine alpha myosin heavy chain gene leads to dosage effects and functional deficits in the heart. J Clin Invest 98:1906-1917

41. Becker KD, Gottshall KR, Hickey R, Perriard JC, Chien KR (1997) Point mutations in human beta cardiac myosin heavy chain have differential effects on sarcomeric structure and assembly: an ATP binding site change disrupts both thick and thin filaments, whereas hypertrophic cardiomyopathy mutations display normal assembly. J Cell Biol 137:131-140

42. Marian AJ, Yu QT, Mann DL, Graham FL, Roberts R (1995) Expression of a mutation causing hypertrophic cardiomyopathy disrupts sarcomere assembly in adult feline cardiac myocytes. Circ Res 77:98-106

43. Marian AJ, Zhao G, Seta Y, Roberts R, Yu QT (1997) Expression of a mutant (Arg92Gln) human cardiac troponin T, known to cause hypertrophic cardiomyopathy, impairs adult cardiac myocyte contractility. Circ Res 81:76-85

44. Rust EM, Albayya FP, Metzger JM (1999) Identification of a contractile deficit in adult cardiac myocytes expressing hypertrophic cardiomyopathy-associated mutant troponin $\mathrm{T}$ proteins. J Clin Invest 103:1459-1467

45. Watkins H, Seidman CE, Seidman JG, Feng HS, Sweeney HL (1996) Expression and functional assessment of a truncated cardiac troponin $\mathrm{T}$ that causes hypertrophic cardiomyopathy. Evidence for a dominant negative action. J Clin Invest 98:2456-2461

46. Westfall MV, Rust EM, Albayya F, Metzger JM (1997) Adenovirus-mediated myofilament gene transfer into adult cardiac myocytes. Methods Cell Biol 52:307-322

47. Metzger JM, Parmacek MS, Barr E, Pasyk K, Lin WI, Cochrane KL, Field LJ, Leiden JM (1993) Skeletal troponin C reduces contractile sensitivity to acidosis in cardiac myocytes from transgenic mice. Proc Natl Acad Sci USA 90:90369040

48. Yang Q, Sanbe A, Osinska H, Hewett TE, Klevitsky R, Robbins J (1999) In vivo modeling of myosin binding protein C familial hypertrophic cardiomyopathy. Circ Res 85:841-847

49. Yang Q, Sanbe A, Osinska H, Hewett TE, Klevitsky R, Robbins J (1998) A mouse model of myosin binding protein C human familial hypertrophic cardiomyopathy. J Clin Invest 102: $1292-1300$

50. Tardiff JC, Hewett TE, Palmer BM, Olsson C, Factor SM, Moore RL, Robbins J, Leinwand LA (1999) Cardiac troponin $\mathrm{T}$ mutations result in allele-specific phenotypes in a mouse model for hypertrophic cardiomyopathy. J Clin Invest 104: 469-481

51. Tardiff JC, Factor SM, Tompkins BD, Hewett TE, Palmer BM, Moore RL, Schwartz S, Robbins J, Leinwand LA (1998) A truncated cardiac troponin $\mathrm{T}$ molecule in transgenic mice suggests multiple cellular mechanisms for familial hypertrophic cardiomyopathy. J Clin Invest 101:2800-2811

52. Vikstrom KL, Factor SM, Leinwand LA (1996) Mice expressing mutant myosin heavy chains are a model for familial hypertrophic cardiomyopathy. Mol Med 2:556-567

53. Muthuchamy M, Pieples K, Rethinasamy P, Hoit B, Grupp IL, Boivin GP, Wolska B, Evans C, Solaro RJ, Wieczorek DF (1999) Mouse model of a familial hypertrophic cardiomyopathy mutation in alpha-tropomyosin manifests cardiac dysfunction. Circ Res 85:47-56

54. Geisterfer-Lowrance AA, Christe M, Conner DA, Ingwall JS, Schoen FJ, Seidman CE, Seidman JG (1996) A mouse model of familial hypertrophic cardiomyopathy. Science 272:731734

55. McConnell BK, Jones KA, Fatkin D, Arroyo LH, Lee RT, Aristizabal O, Turnbull DH, Georgakopoulos D, Kass D, Bond M, Niimura H, Schoen FJ, Conner D, Fischman DA, Seidman CE, Seidman JG, Fischman DH (1999) Dilated cardiomyopathy in homozygous myosin-binding protein-C mutant mice 
[published erratum appears in J Clin Invest 1999 Dec; 104(12): 1771]. J Clin Invest 104:1235-1244

56. Rust EM, Westfall MV, Metzger JM (1998) Stability of the contractile assembly and $\mathrm{Ca} 2+$-activated tension in adenovirus infected adult cardiac myocytes. Mol Cell Biochem 181:143-155

57. Westfall MV, Rust EM, Metzger JM (1997) Slow skeletal troponin I gene transfer, expression, and myofilament incorporation enhances adult cardiac myocyte contractile function. Proc Natl Acad Sci USA 94:5444-5449

58. Redwood CS, Moolman-Smook JC, Watkins H (1999) Properties of mutant contractile proteins that cause hypertrophic cardiomyopathy. Cardiovasc Res 44:20-36

59. Fujita H, Ishiwata S (1999) Tropomyosin modulates pH dependence of isometric tension. Biophys J 77:1540-1546

60. Sweeney HL, Feng HS, Yang Z, Watkins H (1998) Functional analyses of troponin $\mathrm{T}$ mutations that cause hypertrophic cardiomyopathy: insights into disease pathogenesis and troponin function. Proc Natl Acad Sci USA 95:14406-14410

61. Sweeney HL, Feng HS (1998) Structure-function analysis of cytoskeletal/contractile proteins in avian myotubes. Methods Cell Biol 52:275-282

62. Redwood C, Lohmann K, Bing W, Esposito GM, Elliott K, Abdulrazzak H, Knott A, Purcell I, Marston S, Watkins H (2000) Investigation of a truncated cardiac troponin $\mathrm{T}$ that causes familial hypertrophic cardiomyopathy: $\mathrm{Ca}(2+)$ regulatory properties of reconstituted thin filaments depend on the ratio of mutant to wild-type protein. Circ Res 86:1146-1152

63. Michele DE, Albayya FP, Metzger JM (1999) Thin filament protein dynamics in fully differentiated adult cardiac myocytes: toward a model of sarcomere maintenance. J Cell Biol 145:1483-1495

64. Michele DE, Albayya FP, Metzger JM (1999) A nemaline myopathy mutation in alpha-tropomyosin causes defective regulation of striated muscle force production. J Clin Invest 104:1575-1581

65. Michele DE, Albayya FP, Metzger JM (1999) Direct, convergent hypersensitivity of calcium-activated force generation produced by hypertrophic cardiomyopathy mutant alpha-tropomyosins in adult cardiac myocytes. Nature Med 5:1413-1417

66. Fabiato A (1981) Myoplasmic free calcium concentration reached during the twitch of an intact isolated cardiac cell and during calcium-induced release of calcium from the sarcoplasmic reticulum of a skinned cardiac cell from the adult rat or rabbit ventricle. J Gen Physiol 78:457-497
67. Bonow RO (1991) Left ventricular diastolic function in hypertrophic cardiomyopathy. Herz 16:13-21

68. Caputo C, Edman KA, Lou F, Sun YB (1994) Variation in myoplasmic $\mathrm{Ca} 2+$ concentration during contraction and relaxation studied by the indicator fluo-3 in frog muscle fibres. $\mathrm{J}$ Physiol (Lond) 478:137-148

69. Simpson DM, Bender AN (1988) Human immunodeficiency virus-associated myopathy: analysis of 11 patients. Ann Neurol 24:79-84

70. Macpherson PC, Dennis RG, Faulkner JA (1997) Sarcomere dynamics and contraction-induced injury to maximally activated single muscle fibres from soleus muscles of rats. J Physiol (Lond) 500:523-533

71. Karpati G, Carpenter S, Eisen AA (1972) Experimental corelike lesions and nemaline rods: a correlative morphological and physiological study. Arch Neurol 27:237-251

72. Sadoshima J, Izumo S (1997) The cellular and molecular response of cardiac myocytes to mechanical stress. Annu Rev Physiol 59:551-571

73. McKillop DF, Geeves MA (1993) Regulation of the interaction between actin and myosin subfragment 1: evidence for three states of the thin filament. Biophys J 65:693-701

74. Lorenz M, Poole KJ, Popp D, Rosenbaum G, Holmes KC (1995) An atomic model of the unregulated thin filament obtained by X-ray fiber diffraction on oriented actin-tropomyosin gels [published erratum appears in J Mol Biol 1995 Jun 2;249(2):509]. J Mol Biol 246:108-119

75. Lehman W, Rosol M, Hatch V, Korman VL, Horowitz R, VanEyk J, Tobacman LS, Craig R (2000) Tropomyosin control of thin filament activity revealed by electron microscopy and 3-D reconstruction. Biophys J 78:A2356

76. Metzger JM (1995) Myosin binding-induced cooperative activation of the thin filament in cardiac myocytes and skeletal muscle fibers. Biophys J 68:1430-1442

77. Nielsen-Kudsk JE, Aldershvile J (1995) Will calcium sensitizers play a role in the treatment of heart failure? J Cardiovasc Pharmacol 26 [Suppl. 1]:S77-S84

78. Bonow RO, Frederick TM, Bacharach SL, Green MV, Goose PW, Maron BJ, Rosing DR (1983) Atrial systole and left ventricular filling in hypertrophic cardiomyopathy: effect of verapamil. Am J Cardiol 51:1386-1391 